\title{
DEVMA: Developing Virtual Environments with Awareness Models
}

\author{
Pilar Herrero and Angélica de Antonio \\ Facultad de Informática. Universidad Politécnica de Madrid. \\ Campus de Montegancedo S/N. \\ 28.660 Boadilla del Monte. Madrid. Spain \\ \{pherrero, angelica\} @fi.upm.es
}

\begin{abstract}
In this paper, we present an application, called DEVMA, developed at the Universidad Politécnica de Madrid with the aim of introducing into a 2D virtual environment some of the key concepts of the Spatial Model of Interaction (SMI) - an awareness model designed for Computer Supported Collaborative Work (CSCW). This application also takes into account how these concepts can be deformed by the presence of boundaries in the environment and how these deformations could have an influence on the awareness of interaction between them.
\end{abstract}

\section{The Spatial Model of Interaction (SMI)}

The aim of this research was to study how the key concepts of one of the most successful models of awareness in Computer Supported Cooperative Work (CSCW), called the Spatial Model of Interaction (SMI) [1,2], are deformed by the presence of objects that were acting as boundaries in the environment. It allows objects in a virtual world to govern their interaction through some key concepts: medium, aura, awareness, focus, nimbus, adapters and boundaries.

Aura is the sub-space which effectively bounds the presence of an object within a given medium and which acts as an enabler of potential interaction. In each particular medium, it is possible to delimit the observing object's interest. This area is called focus "The more an object is within your focus the more aware you are of it". The focus concept has been implemented in the SMI as an "triangle" cone limited by the object's aura.

In the same way, it is possible to represent the observed object's projection in a particular medium. This area is called nimbus: "The more an object is within your nimbus the more aware it is of you". The nimbus concept, as it was defined in the Spatial Model of Interaction, has always been implemented as an circumference in a visual medium. The radio of this circumference has an "ideal" infinite value, although in practice, it is limited by the object's aura.

The main concept involved in controlling interaction between objects is "awareness". One object's awareness of another object quantifies the subjective importance or relevance of that object. The awareness relationship between every pair of objects is achieved on the basis of quantifiable levels of awareness between them 
and it is unidirectional and specific to each medium. Awareness between objects in a given medium is manipulated via focus and nimbus. Moreover, an object's aura, focus, nimbus, and hence awareness, can be modified through boundaries and some artefacts called adapters.

For a simple discrete model of focus and nimbus, there are tree possible classifications of awareness values when two objects are negotiating unidirectional awareness [3]:

- Full awareness: The awareness that object A has of object B in a medium $\mathrm{M}$ is "full" when object B is inside A's focus and object A is inside B's nimbus (Figure 1).

- Peripheral awareness: The awareness that object A has of object B in a medium M is "peripheral" when (Figure 2) object B is outside A's focus but object A is inside B's nimbus, or object B is inside A's focus but object A is outside B's nimbus.

- No awareness: An object A has no awareness of object B in a medium M when object B is outside A's focus and object A is outside B's nimbus.

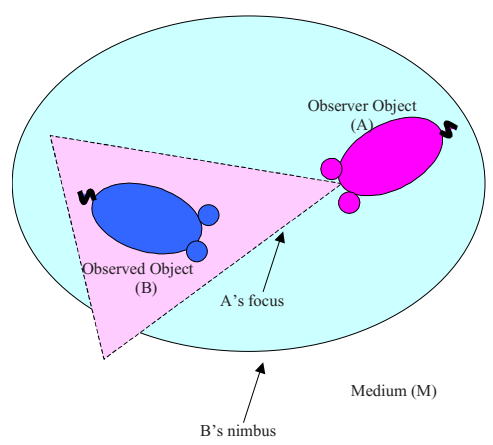

Fig. 1. Full Awareness

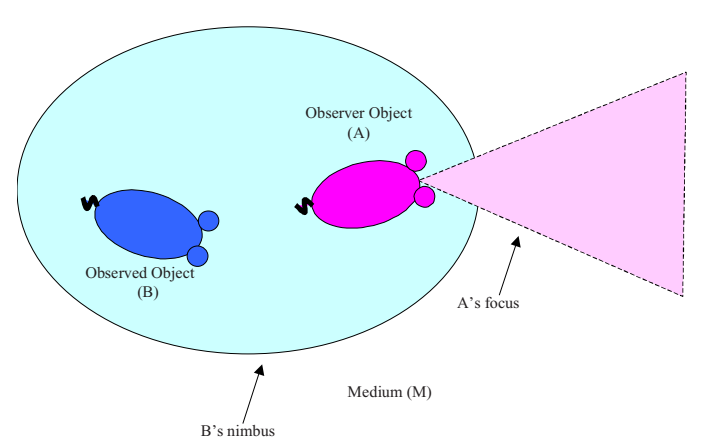

Fig. 2. Peripheral Awareness

In this application we have concentrated on four of these concepts: focus, nimbus, awareness and boundaries. More specifically, we have concentrated on how to introduce the geometrical modifications that boundaries can produce on the focus (and nimbus) shape and how these modifications can have an influence on the awareness of interaction between participants.

\section{The DEVMA Application}

This application shows the avatar's focus and nimbus deformation while it is moving around the environment and interfering with some boundaries. The user can introduce one or more avatars, having the chance of modifying its spatial properties - such as the avatar's position.

The application also allows to modify some of the focus properties such as the focus' length (see "longitud" in the Figure 3) or the focus' angle (see "Ángulo" in the Fig. 3). In the same way, the DEVMA application allows the user to modify the nimbus' ratio [4]. 


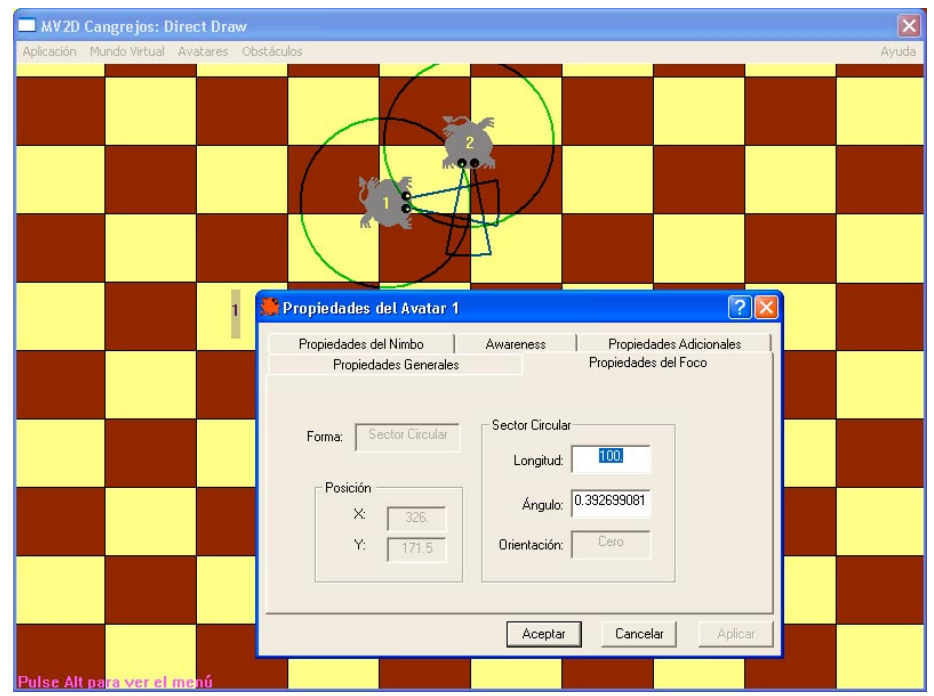

Fig. 3. Focus' Properties

The user also has the possibility of introducing one or more boundaries, interacting with each of them using the keyboard, mouse or application menu. When the avatar's focus (or nimbus) intersect with an obstacle, the focus's (or nimbus') shape will be deformed as it is showed in the figures 4 .

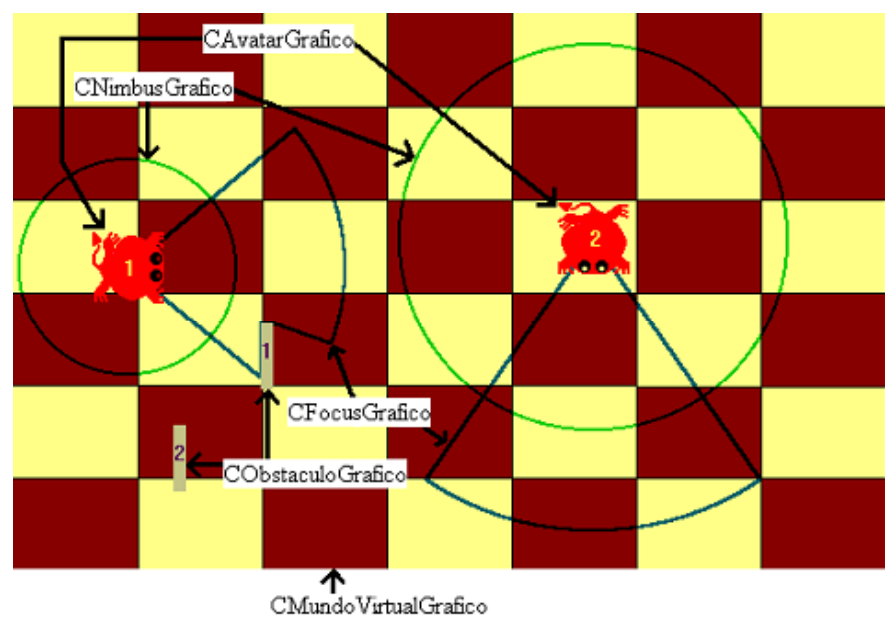

Fig. 4. Avatar's Focus and Nimbus Deformation in the Environment

The application also determine if there is any kind of awareness between avatars and, if later, it can classify it as full awareness, peripheral awareness or no awareness. In the figure 5 it is possible to appreciate how there is a "Full Awareness" between the avatars 1 and 2 . 


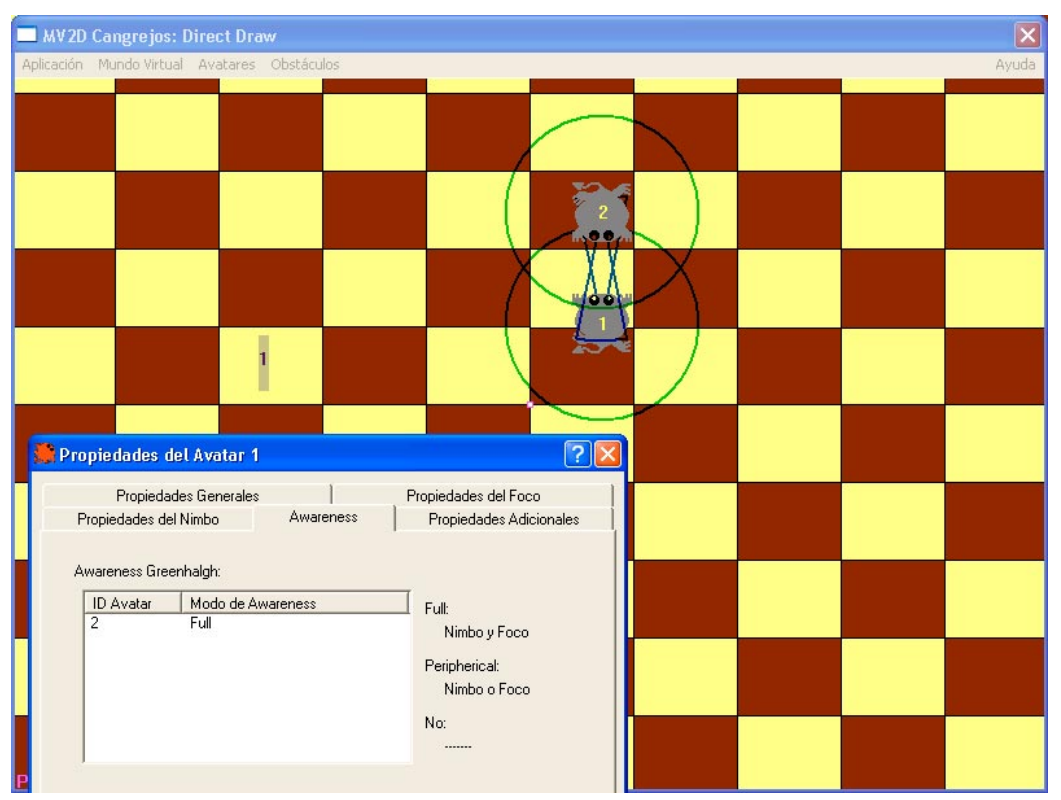

Fig. 5. Full Awareness between Avatars

\section{References}

1. Benford, S. Prinz, W. Mariani, J. Rodden, T. Navarro, L. Bignoli, E. Grant Brown C. and Naslund, T. MOCCA - A Distributed Environment For Collaboration, Available from the MOCCA Working Group of Co-Tech.

2. Benford, S., and Fahlén, L.E. A spatial model of interaction in large virtual environments, in Proc. Third European Conference on Computer Supported Cooperative Work (ECSCW'93), Milano, Italy. Kluwer Academic Publishers, pp. 109-124.

3. Greenhalgh, C., Large Scale Collaborative Virtual Environments, Doctoral Thesis. University of Nottingham. October 1997.

4. Herrero P. A Human-Like Perceptual Model for Intelligent Virtual Agents PhD Thesis. Universidad Politécnica de Madrid, June 2003. 